\title{
Endowments or Returns to Endowments or Both? Deciphering Disparities in Childhood Stunting in Bihar, India
}

\section{CURRENT STATUS: UNDER REVISION}

BMC Nutrition BMC Series

Saswata Ghosh

चhosh.saswata@gmail.comCorresponding Author

ORCiD: https://orcid.org/0000-0001-9337-683X

Santosh Kumar Sharma

Centre for Health Policy, ADRI

Debarshi Bhattacharya

Bill and Melinda Gates Foundation

DOI:

$10.21203 / \mathrm{rs} .2 .18284 / \mathrm{v} 1$

\section{SUBJECT AREAS}

Nutrition \& Dietetics

KEYWORDS

Deciphering, Disparities in Childhood, Stunting in Bihar, India 
Abstract

Background Unacceptably high rate of childhood stunting for decades remained a puzzle in the Indian state of Bihar, located in the eastern part of the country. Despite various programmatic interventions, nearly half of the under-five children (numerically about 10 million) are still stunted in this resourceconstrained state.

Data and Methods Using four successive rounds of National Family Health Survey (NFHS) data spread over more than two decades and employing quantile regressions and counterfactual decomposition (QR-CD), the present study aims to assess effects of various endowments as well as returns to those endowments in disparities in childhood stunting over the period.

Results The results show that although child's height-for-age Z-scores (HAZ) disparity was largely accounted for differing levels of endowments during earlier decade, in the later periods, inadequate access to the benefits from various development programmes was also found responsible for HAZ disparities. Moreover, effects of endowments and their returns vary across quantiles. We argue that apart from equalizing endowments, ensuring adequate access to different nutrition-centric programmes are essential to lessen the burden of childhood stunting.

Conclusion The state must focus on intersectoral convergence of different schemes in the form of state nutrition mission, and, strengthen nutrition-centric policy processes and their political underpinnings to harness better dividend.

\section{Full Text}

Due to technical limitations, full-text HTML conversion of this manuscript could not be completed. However, the manuscript can be downloaded and accessed as a PDF.

\section{Tables}

Tables 


\begin{tabular}{|c|c|c|}
\hline $\begin{array}{l}\text { Demographic and Health } \\
\text { Indicators }\end{array}$ & 1991 & 2015-16 \\
\hline Population('000s) & $8,63,38,853^{a}$ & $10,40,99,452 f$ \\
\hline $\begin{array}{l}\text { Population Density } \\
\text { (person } / \mathrm{km}^{2} \text { ) }\end{array}$ & $685^{a}$ & $1102^{\mathrm{h}}$ \\
\hline Sex ratio (female/1000 male) & $907^{a}$ & $916 \mathrm{~h}$ \\
\hline $\begin{array}{l}\% \text { population aged } 6+\text { that is } \\
\text { literate }\end{array}$ & $38.5^{a}$ & $63.8 \mathrm{~h}$ \\
\hline $\begin{array}{l}\% \text { female population aged } \\
6+\text { that is literate }\end{array}$ & $22.9^{a}$ & $53.3 \mathrm{~h}$ \\
\hline Child (0-6 years) sex ratio & $953^{a}$ & $908 \mathrm{~h}$ \\
\hline $\begin{array}{l}\text { life expectancy at birth (in } \\
\text { years) }\end{array}$ & $57.5^{\mathrm{b}}$ & $68.1 \mathrm{i}$ \\
\hline Infant mortality rate & $69^{c}$ & $42 i$ \\
\hline Total fertility rate & $4.4^{\mathrm{a}}$ & $3.3 \mathrm{i}$ \\
\hline Maternal mortality ratio & $452^{d}$ & $165^{\mathrm{j}}$ \\
\hline $\begin{array}{l}\% \text { mothers who had at least } \\
3 \text { ANC for last birth }\end{array}$ & $30.7^{e}$ & $14.4 \mathrm{k}$ \\
\hline $\begin{array}{l}\% \text { skilled attendance at } \\
\text { delivery }\end{array}$ & $19.0^{\mathrm{e}}$ & $70.0 \mathrm{k}$ \\
\hline$\%$ institutional delivery & $13.0^{\mathrm{e}}$ & $63.8 \mathrm{k}$ \\
\hline Head count poverty ratio & $45.9 \%^{f}$ & $43 \% 1$ \\
\hline Economic growth (GDP) & $4.6^{\mathrm{g}}$ & $10.3 \mathrm{~m}$ \\
\hline $\begin{array}{l}\text { Singulate Mean age at } \\
\text { marriage }\end{array}$ & $18.0^{\mathrm{e}}$ & $19.5 n$ \\
\hline
\end{tabular}

Sources: a Census of India, 2001; b SRS 1988-92; ' NIPCCD (National Institute of Public Cooperation and Child Development 2014; ${ }^{d}$ SRS 2000, figure pertain to 1998; e NFHS 1 (1992-93); ${ }^{f}$ World Bank 1993; g Economic and Political Weekly 2000; ${ }^{\text {h }}$ Census 2011; i SRS 2016; j SRS 2014-16 (Jharkhand

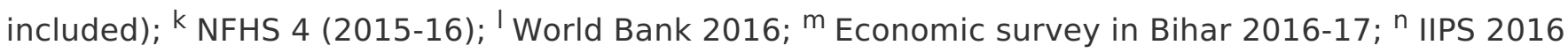




\begin{tabular}{|c|c|c|c|c|c|c|c|}
\hline Bihar & NFHS 1 & NFHS 2 & NFHS 3 & NFHS 4 & $\begin{array}{c}\text { Absolute } \\
\text { increase } \\
\text { btw NFHS } \\
1 \text { and } \\
\text { NFHS } 2\end{array}$ & $\begin{array}{c}\text { Absolute } \\
\text { increase } \\
\text { btw NFHS } \\
2 \text { and } \\
\text { NFHS } 3\end{array}$ & $\begin{array}{c}\text { Absolute } \\
\text { increase } \\
\text { btw NFHS } \\
3 \text { and } \\
\text { NFHS } 4\end{array}$ \\
\hline 10 & -4.92 & -4.89 & -4.03 & -3.74 & 0.03 & 0.86 & 0.29 \\
\hline 25 & -3.85 & -3.75 & -2.95 & -2.81 & 0.10 & 0.80 & 0.14 \\
\hline 50 & -2.49 & -2.46 & -1.93 & -1.80 & 0.03 & 0.54 & 0.13 \\
\hline 75 & -1.09 & -1.08 & -0.84 & -0.61 & 0.01 & 0.24 & 0.23 \\
\hline 90 & 0.12 & 0.36 & 0.19 & 0.65 & 0.24 & -0.17 & 0.46 \\
\hline Overall & -2.36 & -2.28 & -1.89 & -1.63 & 0.07 & 0.40 & 0.26 \\
\hline India & NFHS 1 & NFHS 2 & NFHS 3 & NFHS 4 & $\begin{array}{c}\text { Absolute } \\
\text { increase } \\
\text { btw NFHS } \\
1 \text { and } \\
\text { NFHS } 2 \\
\end{array}$ & $\begin{array}{c}\text { Absolute } \\
\text { increase } \\
\text { btw NFHS } \\
2 \text { and } \\
\text { NFHS } 3 \\
\end{array}$ & $\begin{array}{c}\text { Absolute } \\
\text { increase } \\
\text { btw NFHS } \\
3 \text { and } \\
\text { NFHS } 4 \\
\end{array}$ \\
\hline 10 & -4.26 & -4.19 & -3.72 & -3.49 & 0.07 & 0.47 & 0.24 \\
\hline 25 & -3.16 & -3.10 & -2.70 & -2.48 & 0.06 & 0.40 & 0.22 \\
\hline 50 & -2.00 & -1.96 & -1.63 & -1.43 & 0.04 & 0.33 & 0.20 \\
\hline 75 & -0.85 & -0.82 & -0.51 & -0.27 & 0.03 & 0.31 & 0.24 \\
\hline 90 & 0.32 & 0.31 & 0.64 & 0.99 & -0.01 & 0.33 & 0.35 \\
\hline Overall & -1.94 & -1.91 & -1.55 & -1.30 & 0.03 & 0.36 & 0.25 \\
\hline
\end{tabular}

Source: Computed by the authors from unit-level data of NFHS rounds

\begin{tabular}{|c|c|c|c|c|}
\hline & NFHS 1 & NFHS 2 & NFHS 3 & NFHS 4 \\
\hline Child HAZ (Mean) & $-2.36(0.05)$ & $-2.29(0.05)$ & $-1.89(0.05)$ & $-1.63(0.04)$ \\
\hline $\begin{array}{l}\text { Age of Child in } \\
\text { Month (mean) }\end{array}$ & 16.26 & 16.35 & 17.45 & 17.71 \\
\hline Age2 (mean) & 357.20 & 378.48 & 405.54 & 414.71 \\
\hline Child Sex & & & & \\
\hline Male & 49.5 & 52.1 & 53.45 & 51.8 \\
\hline $\begin{array}{l}\text { Female } \\
\text { Birth Size }\end{array}$ & 50.5 & 47.9 & 46.55 & 48.2 \\
\hline Normal & 71.9 & 69.5 & 47.23 & 69.34 \\
\hline Average and above & 11.0 & 14.5 & 31.5 & 17.42 \\
\hline $\begin{array}{l}\text { Small } \\
\text { Early } \\
\text { Breastfeeding }\end{array}$ & 17.1 & 16.1 & 21.27 & 13.24 \\
\hline
\end{tabular}




\begin{tabular}{|c|c|c|c|c|}
\hline No & 98.2 & 95.6 & 97.39 & 63.39 \\
\hline Yes & 1.8 & 4.4 & 2.61 & 36.61 \\
\hline $\begin{array}{l}\text { No. of Sibling } \\
\text { (mean) }\end{array}$ & 1.88 & 1.96 & 2.06 & 1.62 \\
\hline \multicolumn{5}{|l|}{$\begin{array}{l}\text { Benefitted ICDS } \\
\text { services }\end{array}$} \\
\hline No & - & - & 92.06 & 39.04 \\
\hline Yes & - & - & 7.94 & 60.96 \\
\hline \multicolumn{5}{|l|}{ Mother's } \\
\hline \multicolumn{5}{|l|}{ Characteristics } \\
\hline \multicolumn{5}{|l|}{ Institutional } \\
\hline \multicolumn{5}{|l|}{ Delivery } \\
\hline No & 87.6 & 84.9 & 77.98 & 30.84 \\
\hline Yes & 12.4 & 15.1 & 22.02 & 69.16 \\
\hline $\begin{array}{l}\text { Age of mother at } \\
\text { first birth (mean) }\end{array}$ & 18.55 & 18.28 & 18.51 & 20.42 \\
\hline $\begin{array}{l}\text { BMI of mother } \\
\text { (mean) }\end{array}$ & - & 19.34 & 19.30 & 20.06 \\
\hline \multicolumn{5}{|l|}{$\begin{array}{l}\text { Mother's } \\
\text { anaemia }\end{array}$} \\
\hline No & - & 71.09 & 80.08 & 83.1 \\
\hline Yes & - & 28.91 & 19.2 & 16.9 \\
\hline $\begin{array}{l}\text { Mother's height } \\
\text { (mean) }\end{array}$ & - & 149.6 & 150.01 & 149.5 \\
\hline Mother's & 2.02 & 2.01 & 2.93 & 3.98 \\
\hline \multicolumn{5}{|l|}{ Education (mean) } \\
\hline \multicolumn{5}{|l|}{ Working mother } \\
\hline No & 78.1 & 80.6 & 79.9 & 89.20 \\
\hline Yes & 21.9 & 19.7 & 20.1 & 10.20 \\
\hline Media exposure & $0.47(0-3)$ & $0.43(0-3)$ & $0.60(0-3)$ & $1.31(0-3)$ \\
\hline \multicolumn{5}{|l|}{ Religion } \\
\hline Hindu & 78.2 & 80.4 & 81.57 & 79.24 \\
\hline Muslim/Others & 21.8 & 17.6 & 18.43 & 20.76 \\
\hline \multicolumn{5}{|l|}{ Caste } \\
\hline SC & 9.3 & 23.72 & 19.6 & 19.9 \\
\hline ST & 8.5 & 1.3 & 0.9 & 3.7 \\
\hline Others & 82.2 & 74.98 & 79.6 & 76.4 \\
\hline \multicolumn{5}{|l|}{ Wealth index } \\
\hline Poorest & 20.5 & 18.2 & 31.7 & 55.5 \\
\hline Poorer & 25.9 & 20.4 & 33.3 & 24.8 \\
\hline Middle & 16.9 & 23.3 & 16.6 & 11.6 \\
\hline Richer & 13.8 & 23.2 & 12.5 & 6.3 \\
\hline Richest & 22.9 & 14.9 & 5.8 & 1.8 \\
\hline \multicolumn{5}{|l|}{$\begin{array}{l}\text { Place of } \\
\text { residence }\end{array}$} \\
\hline Urban & 13.1 & 6.2 & 11.1 & 10.8 \\
\hline Rural & 86.9 & 93.8 & 88.9 & 89.2 \\
\hline Total & 1821 & 2029 & 1188 & 2184 \\
\hline
\end{tabular}

Source: Computed by the authors from unit-level data of NFHS rounds 


\begin{tabular}{|c|c|c|c|c|c|c|c|c|c|c|}
\hline & \multicolumn{5}{|c|}{ NFHS 1} & \multicolumn{5}{|c|}{ NFHS 2} \\
\hline & 10 & 25 & 50 & 75 & 90 & 10 & 25 & 50 & 75 & 90 \\
\hline $\begin{array}{l}\text { Age of } \\
\text { Child }\end{array}$ & $\begin{array}{c}-0.073 * * \\
*\end{array}$ & $\begin{array}{c}-0.103 * * \\
*\end{array}$ & $\begin{array}{c}-0.171 * * \\
*\end{array}$ & $\begin{array}{c}-0.173 * * \\
*\end{array}$ & $\begin{array}{c}-0.086 * \\
* *\end{array}$ & 0.025 & $-0.104 *$ & $\begin{array}{c}-0.237 * \\
* *\end{array}$ & $\begin{array}{c}-0.303 * \\
* *\end{array}$ & $\begin{array}{c}-0.419 \\
*\end{array}$ \\
\hline Age2 & $\begin{array}{c}0.001^{* *} \\
*\end{array}$ & $\begin{array}{c}0.001^{* *} \\
*\end{array}$ & $\begin{array}{c}0.003^{* *} \\
*\end{array}$ & $\begin{array}{c}0.003^{* *} \\
*\end{array}$ & $\begin{array}{c}0.001^{* *} \\
*\end{array}$ & -0.003 & 0.000 & $0.004^{* *}$ & $\begin{array}{c}0.006 * * \\
*\end{array}$ & $0.010 *$ \\
\hline Female & $\begin{array}{c}0.234^{* *} \\
*\end{array}$ & $\begin{array}{c}0.306 * * \\
*\end{array}$ & $\begin{array}{c}0.344^{* *} \\
*\end{array}$ & $\begin{array}{c}0.367^{* *} \\
*\end{array}$ & $\begin{array}{c}0.578 * * \\
*\end{array}$ & 0.442 & $0.625^{* *}$ & $\begin{array}{c}1.089 * * \\
*\end{array}$ & 0.412 & 0.480 \\
\hline $\begin{array}{l}\text { Birth } \\
\text { Size } \\
\text { Normal }\end{array}$ & & & & & & & & & & \\
\hline $\begin{array}{l}\text { Averag } \\
\text { e and } \\
\text { above }\end{array}$ & 0.054 & -0.102 & 0.077 & $\begin{array}{c}0.485^{* *} \\
*\end{array}$ & $\begin{array}{c}0.332^{* *} \\
*\end{array}$ & 0.218 & 0.580 & $0.833^{*}$ & 0.944 & 2.332 \\
\hline Small & $\begin{array}{c}-0.185^{* *} \\
*\end{array}$ & -0.003 & 0.066 & 0.002 & 0.088 & -0.505 & 0.416 & 0.476 & 1.127* & -0.291 \\
\hline $\begin{array}{l}\text { Early } \\
\text { Breastf } \\
\text { eeding } \\
\text { (Yes) }\end{array}$ & $\begin{array}{c}0.820 * * \\
*\end{array}$ & $\begin{array}{c}1.063^{* *} \\
*\end{array}$ & $\begin{array}{c}0.447^{* *} \\
*\end{array}$ & $\begin{array}{c}0.422^{* *} \\
*\end{array}$ & -0.242 & -1.281 & -1.137 & -0.117 & -0.064 & $\begin{array}{c}-2.467 \\
*\end{array}$ \\
\hline $\begin{array}{l}\text { No. of } \\
\text { Sibling } \\
\text { Mother } \\
\text { 's } \\
\text { Charac } \\
\text { teristic } \\
\text { s }\end{array}$ & -0.003 & 0.007 & $-0.017^{*}$ & -0.013 & $0.036 * *$ & -0.089 & -0.014 & 0.109 & 0.146 & 0.158 \\
\hline $\begin{array}{l}\text { Instituti } \\
\text { onal } \\
\text { Deliver } \\
y \text { (yes) }\end{array}$ & $\begin{array}{c}0.398 * * \\
*\end{array}$ & $\begin{array}{c}0.888^{* *} \\
*\end{array}$ & $\begin{array}{c}0.398^{* *} \\
*\end{array}$ & $0.135^{* *}$ & $\begin{array}{c}-0.192 * \\
*\end{array}$ & 0.472 & $1.68 * * *$ & $2.231^{* *}$ & 1.943 & 4.823 \\
\hline $\begin{array}{l}\text { Age of } \\
\text { mother } \\
\text { at first } \\
\text { birth }\end{array}$ & $\begin{array}{c}-0.021 * * \\
*\end{array}$ & 0.005 & -0.004 & $\begin{array}{c}0.020 * * \\
*\end{array}$ & $\begin{array}{c}0.051 * * \\
*\end{array}$ & 0.061 & 0.018 & 0.045 & 0.000 & -0.006 \\
\hline $\begin{array}{l}\text { BMI of } \\
\text { mother } \\
\text { Mother } \\
\text { 's } \\
\text { anaem } \\
\text { ia }\end{array}$ & & & & & & 0.000 & 0.000 & $\begin{array}{c}0.001 * * \\
*\end{array}$ & 0.001 & 0.003 \\
\hline Yes & - & - & - & - & - & 0.258 & -0.274 & $\begin{array}{c}-0.998 * \\
* *\end{array}$ & $\begin{array}{c}-1.256 * \\
* *\end{array}$ & $\begin{array}{c}-2.049 \\
*\end{array}$ \\
\hline $\begin{array}{l}\text { Mother' } \\
\text { s height }\end{array}$ & & & & & & $0.006 * *$ & 0.003 & $0.005^{*}$ & 0.006* & -0.003 \\
\hline $\begin{array}{l}\text { Mother' } \\
\text { s } \\
\text { Educati }\end{array}$ & $\begin{array}{c}0.019 * * \\
*\end{array}$ & 0.003 & $\begin{array}{c}0.025^{* *} \\
*\end{array}$ & $0.03 * * *$ & $0.025^{* *}$ & -0.003 & -0.047 & -0.1 & $\begin{array}{c}-0.187 * \\
*\end{array}$ & -0.213 \\
\hline
\end{tabular}




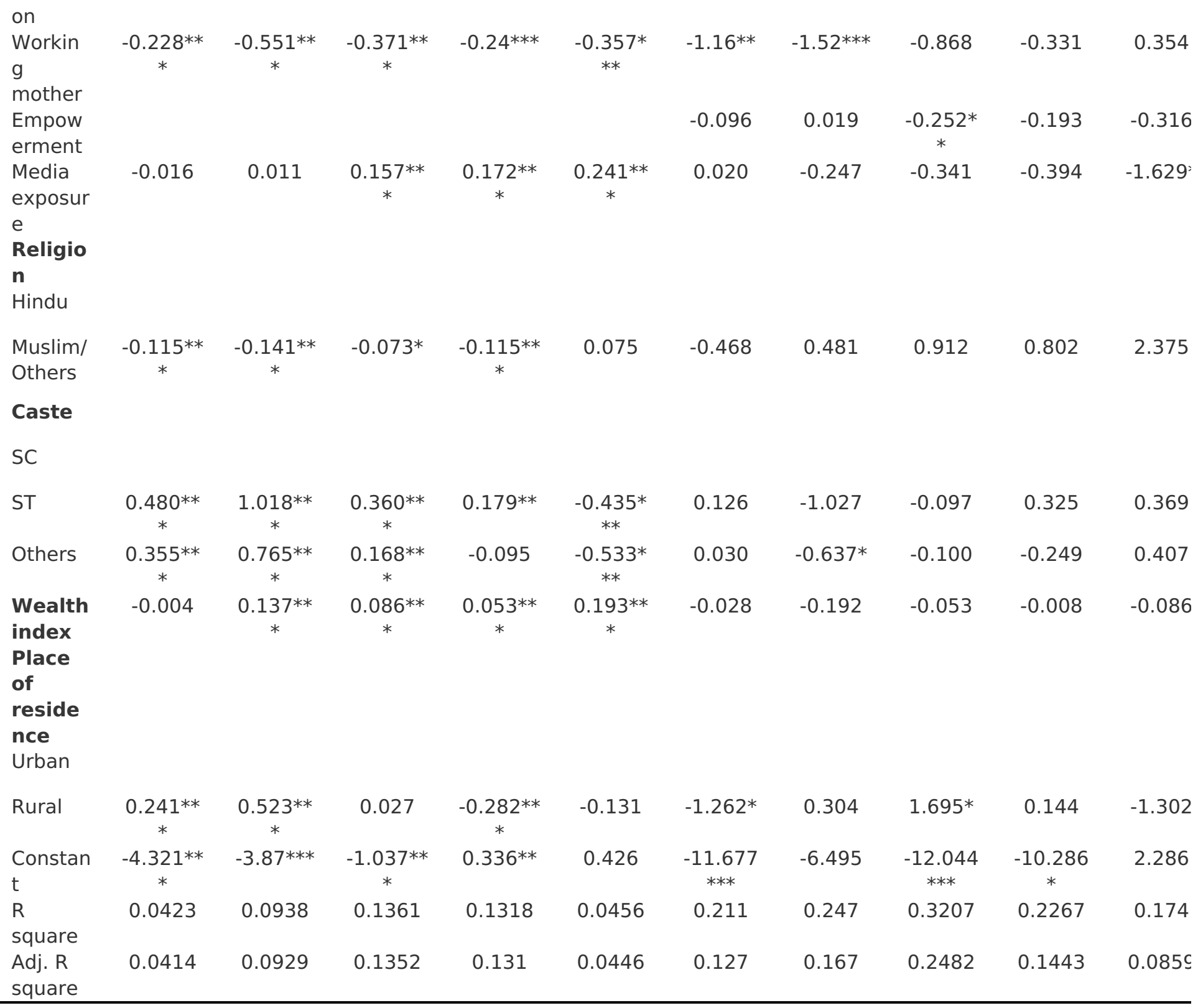

*** $\mathbf{p}<0.001 ; * * \mathbf{p}<0.01 ; * \mathbf{p}<0.05$; Source: Computed by the authors from unit-level data of NFHS 2 
Table 5: Unconditional Re-centred Influence Function (RIF) quantile regression results for NFHS 3 (2005NFHS 4 (2015-16) in Bihar

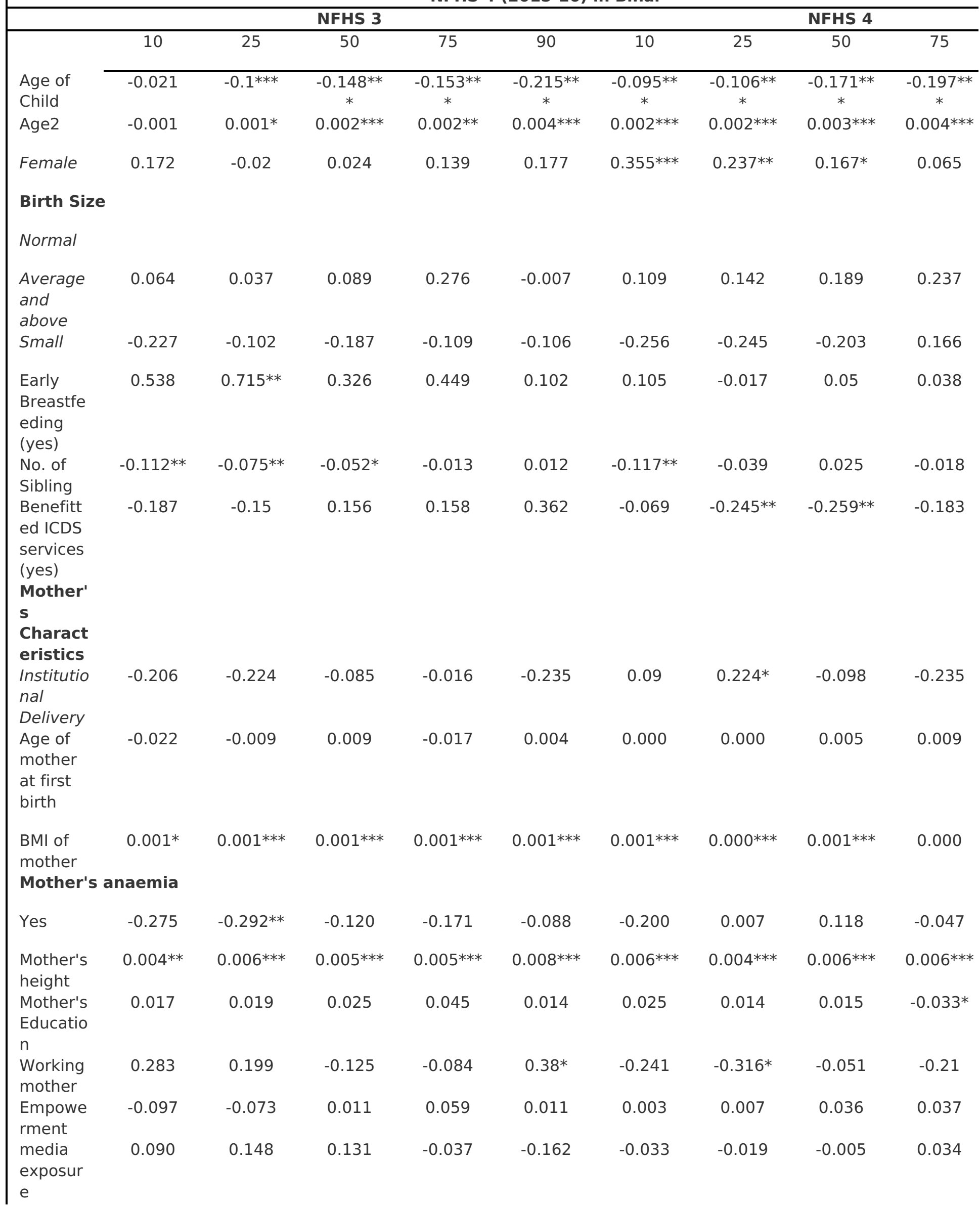




\begin{tabular}{|c|c|c|c|c|c|c|c|c|c|}
\hline \multicolumn{10}{|l|}{$\begin{array}{l}\text { Religio } \\
\mathbf{n} \\
\text { Hindu }\end{array}$} \\
\hline $\begin{array}{l}\text { Muslim/ } \\
\text { Others } \\
\text { Caste }\end{array}$ & 0.187 & -0.015 & 0.108 & 0.286 & -0.290 & 0.035 & -0.099 & -0.118 & $-0.407^{* *}$ \\
\hline \multicolumn{10}{|l|}{ SC } \\
\hline ST & $1.489 * * *$ & 0.427 & 0.15 & -0.094 & -0.429 & 0.249 & 0.023 & 0.087 & -0.065 \\
\hline Others & 0.069 & 0.259 & $0.319 * *$ & $0.529 * * *$ & $0.309 *$ & 0.305 & 0.146 & $0.369 * *$ & 0.16 \\
\hline $\begin{array}{l}\text { Wealth } \\
\text { index } \\
\text { Place of }\end{array}$ & $\begin{array}{l}0.000 * * \\
\text { sidence }\end{array}$ & $0.000 * *$ & $0.000 * *$ & $0.000 * *$ & 0.000 & 0.000 & 0.000 & $0.000 * *$ & $0.000 * *$ \\
\hline \multicolumn{10}{|l|}{ Urban } \\
\hline Rural & 0.207 & 0.023 & 0.006 & 0.265 & 0.085 & -0.177 & -0.089 & -0.132 & -0.173 \\
\hline Constant & $\begin{array}{c}-9.868 * * \\
*\end{array}$ & $\begin{array}{c}-12.311 * \\
* *\end{array}$ & $\begin{array}{c}-9.598 * * \\
*\end{array}$ & $\begin{array}{c}-9.526 * * \\
*\end{array}$ & $\begin{array}{c}-11.783 * \\
* *\end{array}$ & $\begin{array}{c}-12.537 * \\
* *\end{array}$ & $\begin{array}{c}-8.243^{* *} \\
*\end{array}$ & $\begin{array}{c}-9.426 * * \\
*\end{array}$ & $\begin{array}{c}-7.241 * * \\
*\end{array}$ \\
\hline R square & 0.090 & 0.171 & 0.209 & 0.176 & 0.124 & 0.061 & 0.094 & 0.177 & 0.115 \\
\hline $\begin{array}{l}\text { Adj. R } \\
\text { square }\end{array}$ & 0.072 & 0.155 & 0.193 & 0.159 & 0.107 & 0.049 & 0.083 & 0.167 & 0.104 \\
\hline
\end{tabular}

*** $p<0.001 ; * * p<0.01 ; * p<0.05$; Source: Computed by the authors from unit-level data of NFHS 3 and NFHS 4 


\begin{tabular}{|c|c|c|c|c|c|c|c|c|c|c|}
\hline \multicolumn{2}{|c|}{ Table 6: Oaxaca Blinder decomposition of HAZ scores of NFHS 1 and NFHS 2 in Bihar } & 10 & & 25 & & 50 & & 75 & & \\
\hline NFHS $1 \mathrm{H}$ & Z score & $\begin{array}{c}-4.935^{* *} \\
*\end{array}$ & & $\begin{array}{c}-3.870 * * \\
*\end{array}$ & & $\begin{array}{c}-2.511 * * \\
*\end{array}$ & & $\begin{array}{c}-1.160 * * \\
*\end{array}$ & & \\
\hline NFHS $2 \mathrm{H}$ & Z score & $\begin{array}{c}-4.812 * * \\
*\end{array}$ & & $\begin{array}{c}-3.659 * * \\
*\end{array}$ & & $\begin{array}{c}-2.339 * * \\
*\end{array}$ & & $\begin{array}{c}-0.930 * * \\
*\end{array}$ & & 0 \\
\hline $\begin{array}{l}\text { Observed } \\
\text { in } \mathrm{HAZ} \mathrm{sC}\end{array}$ & $\begin{array}{l}\text { Raw gap } \\
\text { res }\end{array}$ & -0.124 & & $\begin{array}{c}-0.211 * \\
* *\end{array}$ & & $\begin{array}{c}-0.172 * \\
*\end{array}$ & & $\begin{array}{c}-0.230 * \\
*\end{array}$ & & $-C$ \\
\hline Covariate & effect & $-0.059 *$ & & -0.050 & & $-0.108 * *$ & & $\begin{array}{c}-0.114 * * \\
*\end{array}$ & & \\
\hline (\% contrib & ution) & 47.7 & & 23.7 & & 62.9 & & 49.6 & & \\
\hline Coefficien & Effect & -0.065 & & -0.161 & & -0.064 & & -0.116 & & -( \\
\hline (\%contrib & tion) & 52.3 & & 76.3 & & 37.1 & & 50.4 & & \\
\hline \multicolumn{6}{|c|}{ Covariate effect } & \multicolumn{5}{|c|}{ Co-efficient effect } \\
\hline & 10 & 25 & 50 & 75 & 90 & 10 & 25 & 50 & 75 & \\
\hline $\begin{array}{l}\text { Aggrega } \\
\text { te effect }\end{array}$ & $-0.059 *$ & -0.050 & $-0.108 * *$ & $\begin{array}{c}-0.114^{* *} \\
*\end{array}$ & -0.106 & -0.065 & $-0.161 *$ & -0.064 & -0.116 & $\overline{-1}$ \\
\hline $\begin{array}{l}\text { Child } \\
\text { Charact } \\
\text { eristics }\end{array}$ & $\begin{array}{c}-0.193 * * \\
*\end{array}$ & $\begin{array}{c}-0.260 * * \\
*\end{array}$ & $\begin{array}{c}-0.261 * * \\
*\end{array}$ & $\begin{array}{c}-0.255^{* *} \\
*\end{array}$ & -0.088 & 0.389 & 0.195 & 0.134 & $-0.818 * *$ & \\
\hline$(\%)$ & 142.1 & 270.8 & 112.7 & 95.6 & 36.5 & -949.8 & -263.9 & -192.1 & 534.7 & \\
\hline $\begin{array}{l}\text { Mother's } \\
\text { Charact } \\
\text { eristics }\end{array}$ & $-0.032 * *$ & $-0.037 * *$ & $-0.033^{*}$ & -0.010 & 0.021 & -0.204 & -0.240 & -0.116 & 0.117 & \\
\hline$(\%)$ & 23.6 & 38.4 & 14.1 & 3.6 & -8.8 & 498.0 & 324.5 & 166.3 & -76.4 & \\
\hline $\begin{array}{l}\text { Househ } \\
\text { old's } \\
\text { Charact } \\
\text { eristics }\end{array}$ & $0.105 *$ & $0.235^{* * *}$ & 0.064 & -0.021 & -0.186 & -0.102 & -0.059 & -0.162 & -0.021 & \\
\hline$(\%)$ & -77.5 & -244.6 & -27.4 & 7.8 & 77.2 & 247.9 & 79.6 & 231.6 & 13.6 & \\
\hline $\begin{array}{l}\text { Spatial } \\
\text { Charact } \\
\text { eristics }\end{array}$ & -0.016 & $-0.034 * *$ & -0.002 & 0.019 & 0.012 & 0.419 & -0.110 & -0.184 & -0.081 & \\
\hline$(\%)$ & 11.9 & 35.8 & 0.9 & -7.1 & -4.8 & -1022.3 & 148.0 & 262.3 & 52.9 & \\
\hline $\begin{array}{l}\text { Constan } \\
\mathrm{t}\end{array}$ & & & & & & -0.543 & 0.139 & 0.258 & 0.649 & \\
\hline $\begin{array}{l}\text { Residual } \\
\mathrm{s}\end{array}$ & 0.077 & 0.047 & 0.124 & 0.153 & 0.135 & -0.024 & -0.087 & 0.006 & 0.037 & \\
\hline Total & -0.136 & -0.096 & $-0.232 * *$ & $\begin{array}{c}-0.267 * * \\
*\end{array}$ & -0.241 & -0.041 & -0.074 & -0.07 & -0.153 & \\
\hline
\end{tabular}

***p<0.001; **p<0.01; *p<0.05; Source: Computed by the authors from unit-level data of NFHS 1 and NFHS 2 


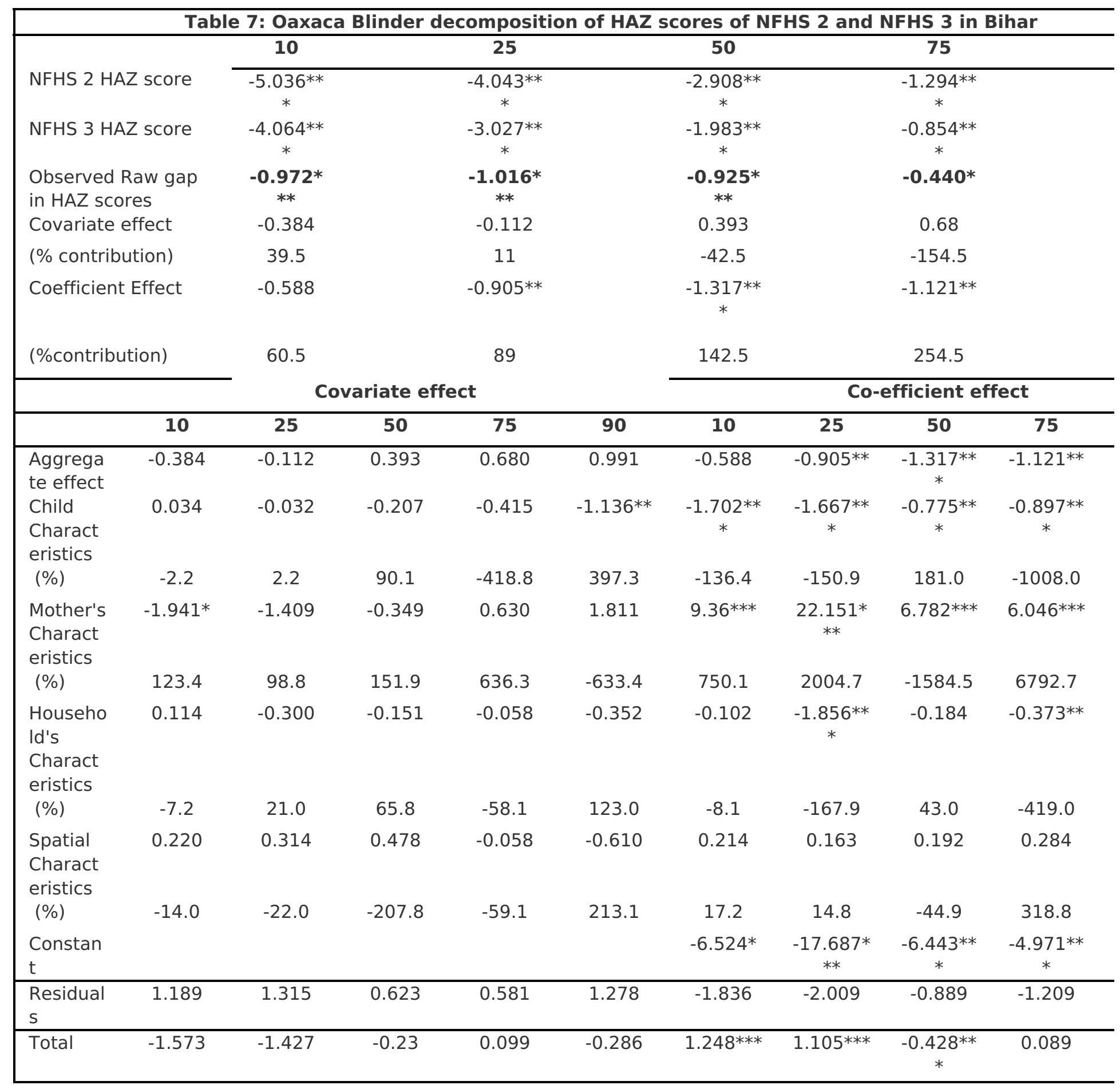

${ }^{* * *} p<0.001 ; * * p<0.01 ; * p<0.05$; Source: Computed by the authors from unit-level data of NFHS 2 and NFHS 3 


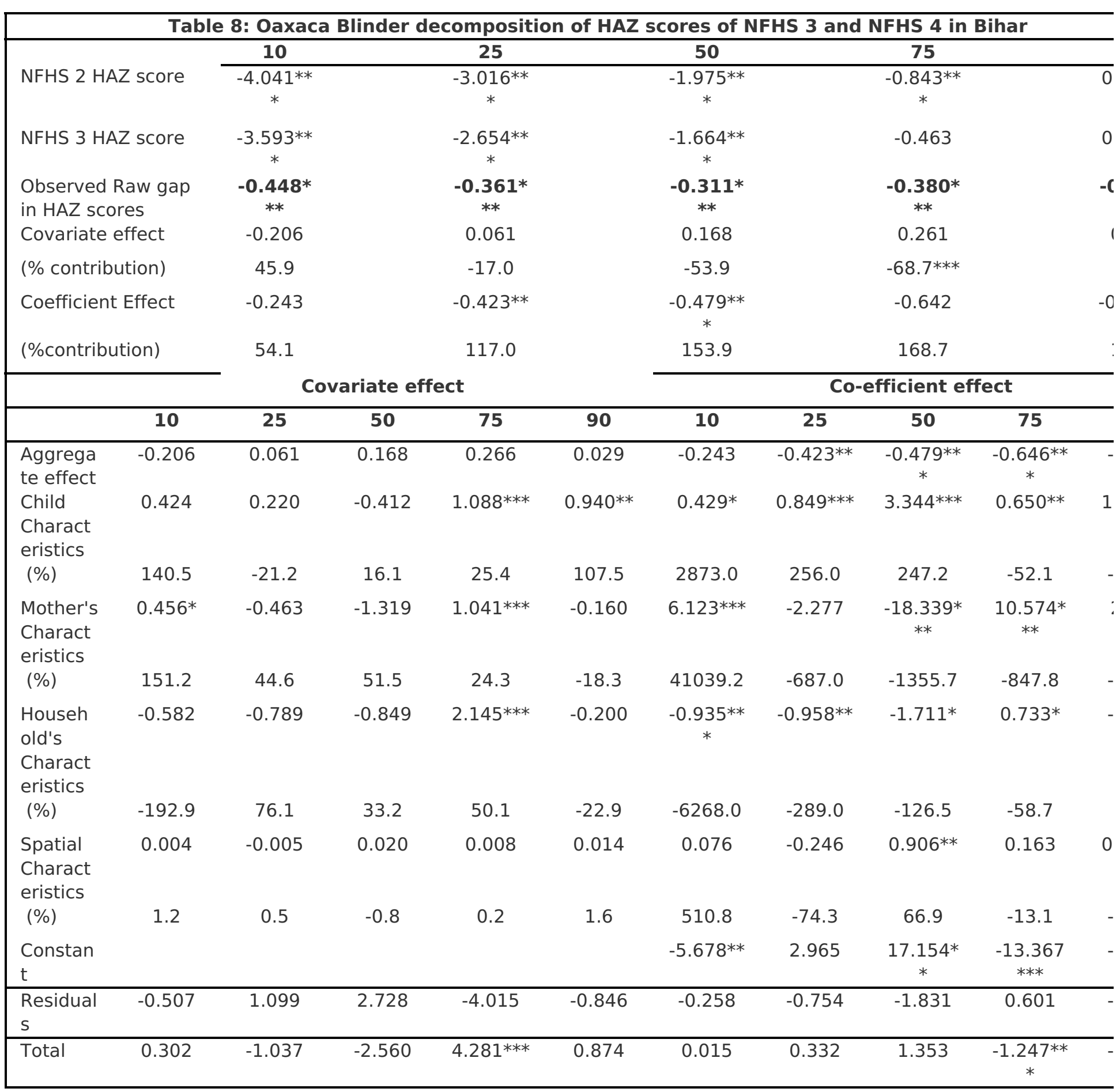

$* * * p<0.001 ; * * p<0.01 ; * p<0.05$; Source: Computed by the authors from unit-level data of NFHS 3 and NFHS 4 


\section{Supplementary Files}

This is a list of supplementary files associated with this preprint. Click to download. appendix.docx 